\title{
FINITE RANK PERTURBATIONS AND SOLUTIONS TO THE OPERATOR RICCATI EQUATION
}

\author{
JULIAN P. GROSSMANN
}

Abstract. We consider an off-diagonal self-adjoint finite rank perturbation of a self-adjoint operator in a complex separable Hilbert space $\mathfrak{H}_{0} \oplus \mathfrak{H}_{1}$, where $\mathfrak{H}_{1}$ is finite dimensional. We describe the singular spectrum of the perturbed operator and establish a connection with solutions to the operator Riccati equation. In particular, we prove existence results for solutions in the case where the whole Hilbert space is finite dimensional.

Mathematics subject classification (2010): Primary 47A62, 47A55; Secondary 47B15. Keywords and phrases: Operator Riccati equation, singular spectrum, Herglotz functions.

\section{REFERENCES}

[1] N. I. Achiezer And I. M. Glazman, Theory of Linear Operators in Hilbert Space, New York: Dover Publications, 1993.

[2] V. Adamyan, H. LANGer, AND C. TRetTER, Existence and uniqueness of contractive solutions of some Riccati equations, in: J. Funct. Anal. 179 (2001), pp. 448-473.

[3] S. Albeverio, K. A. MaKarov, And A. K. Motovilov, Graph subspaces and the spectral shift function, in: Canad. J. Math. 55 (2003), pp. 449-503.

[4] F. Gesztesy and E. TseKanovskit, On matrix-valued Herglotz functions, in: Mathematische Nachrichten 218 (2000), pp. 61-138.

[5] T. Kato, Perturbation Theory for Linear Operators, Berlin: Springer, 1966.

[6] L. Grubišić, V. Kostrykin, K. A. Makarov, And K. Veselić, The Tan $2 \Theta$ theorem for indefinite quadratic forms, in: Journal of Spectral Theory 3 (2013), pp. 83-100. DOI: 10.4171/JST/38.

[7] V. Kostry Kin and K. A. MaKarov, The Singularly Continuous Spectrum and Non-Closed Invariant Subspaces, in: Recent Advances in Operator Theory and its Applications, ed. by I. Gohberg, D. Alpay, J. Arazy et al. Vol. 160, Operator Theory: Advances and Applications, Basel: BirkhäuserVerlag, 2005, pp. 299-309. DOI: 10.1007/3-7643-7398-9_14.

[8] V. Kostrykin, K. A. Makarov, And A. K. Motovilov, Existence and uniqueness of solutions to the operator Riccati equation. A geometric approach, in: Contemporary Mathematics 327 (2003), ed. by Y. Karpeshina, G. Stolz, R. Weikard, Y. Zeng, pp. 181-198. DOI: 10.1090/conm/327/05814.

[9] V. Kostry Kin, K. A. MaKarov, And A. K. Motovilov, A generalization of the tan $2 \Theta$ theorem, in: Current Trends in Operator Theory and Its Applications, Ed. by J. A. Ball, M. Klaus, J. W. Helton, and L. Rodman, vol. 149, Operator Theory: Advances and Applications, Basel: Birkhäuser-Verlag, 2004, pp. 349-372.

[10] P. Lancaster and L. Rodman, Algebraic Riccati Equations, Oxford: University Press, 1995.

[11] H. LANGer AND C. TRETTER, Diagonalization of certain block operator matrices and applications to Dirac operators, in: Operator Theory: Adv. Appl. 122 (2001), pp. 331-358.

[12] H. LANGER, A. C. M. RAN, AND B. A. VAN DE ROTTEN, Invariant subspaces of infinite dimensional Hamiltonians and solutions of the corresponding Riccati equations, in: Linear Operators and Matrices, ed. by I. Gohberg and H. Langer, vol. 130, Operator Theory: Advances and Applications, Basel: Birkhäuser-Verlag, 2002, pp. 235-254.

[13] K. A. MaKarov and A. Seelmann, The length metric on the set of orthogonal projections and new estimates in the subspace perturbation problem, in: Journal für die reine und angewandte Mathematik (Crelles Journal) (2013), DOI: 10.1515/crelle-2013-0099. 
[14] K. A. Makarov, S. Schmitz, And A. Seelmann, On invariant graph subspaces, eprint: arXiv:1509.07984v1 [math.SP] (2015).

[15] W. Rudin, Real and Complex Analysis, International Edition, London: McGraw-Hill, 1987.

[16] C. TRETTER, Spectral theory of block operator matrices and applications, London: Imperial College Press London, 2008.

[17] M. WeISs, Riccati equation theory for Pritchard-Salamon systems: a Popov function approach, Distributed parameter systems: analysis, synthesis and applications, Part 1, in: IMA J. Math. Control Inform. 14 (1997), pp. 45-83. 